\title{
Follow-Up and Risk Assessment in Patients with Myocardial Infarction Using Artificial Neural Networks
}

\author{
Tatjana Gligorijevićc, ${ }^{1,2}$ Zoran Ševarac, ${ }^{3}$ Branislav Milovanović,,${ }^{1,2}$ Vlado Đajić, ${ }^{4}$ \\ Marija Zdravković, ${ }^{1,2}$ Saša Hinić, ${ }^{1}$ Marina Arsić, ${ }^{1}$ and Milica Aleksić ${ }^{1}$ \\ ${ }^{1}$ Department of Cardiology, University Hospital Medical Center Bežanijska Kosa, 11080 Belgrade, Serbia \\ ${ }^{2}$ Medical Faculty, University of Belgrade, 11000 Belgrade, Serbia \\ ${ }^{3}$ Faculty of Organizational Sciences, University of Belgrade, 11000 Belgrade, Serbia \\ ${ }^{4}$ Department of Neurology, University Clinical Center of the Republic of Srpska, 78000 Banjaluka, Bosnia and Herzegovina \\ Correspondence should be addressed to Zoran Ševarac; sevarac@gmail.com
}

Received 24 April 2017; Revised 27 July 2017; Accepted 16 August 2017; Published 17 September 2017

Academic Editor: Gaetano Valenza

Copyright (C) 2017 Tatjana Gligorijević et al. This is an open access article distributed under the Creative Commons Attribution License, which permits unrestricted use, distribution, and reproduction in any medium, provided the original work is properly cited.

\begin{abstract}
Artificial neural networks (ANNs) are machine learning technique, inspired by the principles found in biological neurons. This technique has been used for prediction and classification problems in many areas of medical signal processing. The aim of this paper was to identify individuals with high risk of death after acute myocardial infarction using ANN. A training dataset for ANN was 1705 consecutive patients who underwent 24-hour ECG monitoring, short ECG analysis, noninvasive beat-to-beat heart-rate variability, and baroreflex sensitivity that were followed for 3 years. The proposed neural network classifier showed good performance for survival prediction: $88 \%$ accuracy, $81 \%$ sensitivity, $93 \%$ specificity, $0.85 F$-measure, and area under the curve value of 0.77 . These findings support the theory that patients with high sympathetic activity (reduced baroreflex sensitivity) have an increased risk of mortality independent of other risk factors and that artificial neural networks can indicate the individuals with a higher risk.
\end{abstract}

\section{Introduction}

Many studies have been published on the subject of cardiac risk assessment, with the goal to identify the subgroup of patients who are at high risk of death after myocardial infarction (MI) [1]. The identification of those individuals which are in higher risk of developing the second cardiovascular event is very important so that they can be properly followed and treated. Contemporary cardiac rehabilitation/secondary prevention (CR/SP) programs are designed to reduce cardiovascular risk and event rates, foster healthy behaviors, and promote active lifestyles [2]. The exceedingly difficult challenge for cardiologists is determining which patients are at sufficiently high risk of an arrhythmic death. Therefore it is essential to identify individuals with arrhythmogenic background who will derive benefit from an implantable cardioverter-defibrillators (ICD), in terms of primary prevention of sudden cardiac death (SCD) based on heart-rate variability analysis [3]. In previous studies heartrate variability parameters are proved to be independent risk predictors of SCD after MI [4]. Despite all this knowledge risk stratification models have limited predictive accuracy. Recently published studies suggest that more than half of all implanted primary prevention devices have not delivered any shocks before battery replacement is required [5]. Precise classification and accurate predictive model can improve clinical pathways of the most vulnerable patients. The predictive power of used statistical models is limited, so the alternative models have arisen, and artificial neural networks (ANNs) become more popular [6].

The ANN presents a machine learning technique that could potentially improve performance of predicting clinical outcomes. Artificial neural networks are excellent solution for classifiers with multiple input parameters and pattern recognition problems [7]. The ANN can predict a specific category of a set of input variables which can be further 
used for detailed analysis. The previous studies failed to detect the particular prognostic accuracy in risk assessment after uncomplicated myocardial infarction [8]. To limit the emphasis on incidental associations between input variables, it might be beneficial to guide artificial neural network development by presenting a subset of input variables that are more likely to predict an outcome. This paper deals with the classification and risk assessment of different autonomic nervous system groups based on certain parameters of heartrate variability using an artificial neural network (ANN). The main motivation in our study for using ANN was their ability to solve the highly nonlinear problems and discover unknown relationships between input parameters. The first aim of the study was to improve the prediction of death using different prediction techniques, like ANN and addition to standard statistical models in early phase after acute myocardial infarction. The second goal was identification of those individuals with higher cardiovascular risk from all-cause mortality. Last but not least, the problem which should be pointed out is the methodology of preparing data, variable selection, determination of cutoff values for suggested variables, and making an accurate model for prediction. The methodology and early results of this study were presented and discussed at The 7th International Symposium on Noninvasive Electrocardiology [9].

Determination of individuals with high cardiovascular risk in the acute phase after myocardial infarction using autonomic nervous system patterns and previously wellestablished independent risk predictors was improved using ANN $[10,11]$.

The recognition of variable patterns correlating with an adverse outcome contributed to the understanding of the autonomic background and ability of ANN to "learn" complex relationships between a series of input (predictor) variables and the corresponding output (outcome) variables [12]. Based on relationships identified between input and output variables, trained dataset can be used for pattern recognition or classification tasks in a separate test dataset. Commonly used statistical models use linear combinations of variables and, therefore, are not adept at modeling grossly nonlinear complex interactions as has been demonstrated in complex cardiovascular system [13]. In previously conducted studies some of the advantages of ANN over standard statistical methods have been suggested: neural network models require less formal statistical training to develop, can implicitly detect complex nonlinear relationships between independent and dependent variables, have the ability to detect all possible interactions between predictor variables, and can be developed using multiple different training algorithms [12]. ANN has been successfully applied to a broad range of biomedical problems, and previous studies have demonstrated that various ANN approaches can accurately predict an outcome [1417].

\section{Material and Methods}

2.1. Study Population. The patient population used for ANN training consisted of 1705 patients admitted between 2003 and 2013 to Coronary Care Unit of Clinical Hospital Center
Bežanijska Kosa, Belgrade, Serbia. The admitted patients were recorded the first day after acute myocardial infarction using the electrocardiogram (ECG) and short-term HRV analysis, while Holter ECG was recorded after two weeks. Date of the first visit was taken as the start date, and survival status was determined by contacting the patient. Survival data were used to assess their hazard ratio and to determine all-cause mortality.

All experimental protocols were approved by the local Scientific Ethical Committee of University Clinical Center "Bežanijska Kosa," license number 1039/3. All the participants were fully informed about the study and gave their written consent in agreement with the Declaration of Helsinki.

Inclusion criteria were man $>40$ years old or postmenopausal women, acute ischemic heart disease verified by cardiac biochemical markers, ST-segment depression, or T-wave inversion $\geq 0.1 \mathrm{mV}$ in at least two contiguous leads without the presence of concomitant Q-waves in these leads.

Exclusion criteria were left ventricular hypertrophy, right ventricular hypertrophy, right and left bundle branch block, atrial flutter and fibrillation, anterior and posterior hemiblock, paced rhythm, Wolff-Parkinson-White syndrome, and ventricular tachycardia or incomplete or erroneous data.

2.2. Database. Data were obtained using short ECG analysis (Shiller AT-10), noninvasive beat-to-beat heart-rate variability, and baroreflex sensitivity (Task Force Monitor) and 24hour ambulatory ECG monitoring with long-term HRV analysis. ECG parameters were obtained from a 12-channel recording over the past 5 minutes using the commercial software (Schiller AT-10, Austria). The Task Force Monitor (CNSystems, Graz, Austria) was used to monitor beat-tobeat heart rate by ECG and beat-to-beat blood pressure by the vascular unloading technique [18], which was corrected automatically to the oscillometric blood pressure measured on the contralateral arm. The Task Force Monitor automatically provides the beat-to-beat spectral analysis of heart rate and systolic and diastolic blood pressure variability, applying an autoregressive methodology. Baroreceptor reflex sensitivity (BRS) was automatically assessed using the sequence technique according to Parati et al. [19]. Intervention marks can be set using the Task Force Monitor; it helps define periods for automated statistical analysis [20]. The mean value and standard deviation (SD) of the measured parameters were computed automatically for defined steady-state period. Twenty-four-hour ambulatory ECG recordings were obtained by a 12-lead electrocardiogram, sampling rate of $1000 \mathrm{~Hz}$ per each lead (Cardioscan, DMS, USA), and analyzed by an experienced analyst. The records were screened, corrected, and readied for further analysis. Analysis of the frequency (spectral) domain parameters was performed using Fast Fourier Transformation (FFT) and Hanning window.

2.3. Preprocessing. During the analysis of the gathered data, several problems were identified: extreme values, missing values, and unbalanced data. Extreme values are result of the errors during recording (patient movement, poor connection, etc.) and they were removed manually to be sure 
TABLE 1: Detailed description of input variables used in this study.

\begin{tabular}{|c|c|c|c|}
\hline Methods & Parameters & Units & Description \\
\hline \multirow{5}{*}{ ECG parameters } & QT interval & $\mathrm{ms}$ & $\begin{array}{l}\text { Measure of the time between the start of the Q wave and the end of } \\
\text { the T wave }\end{array}$ \\
\hline & QTc interval & $\mathrm{ms}$ & Heart rate corrected QT interval \\
\hline & PR interval & $\mathrm{ms}$ & $\begin{array}{l}\text { Measure of time from the beginning of the upslope of the } \mathrm{P} \text { wave to } \\
\text { the beginning of the QRS wave }\end{array}$ \\
\hline & QRS complex & $\mathrm{ms}$ & Complex of $\mathrm{Q}$ wave, $\mathrm{R}$ wave, and $\mathrm{S}$ wave \\
\hline & P wave & $\mathrm{ms}$ & The $\mathrm{P}$ wave is the first positive deflection on the ECG \\
\hline \multirow{4}{*}{ Time domain analysis } & Average HR & bpm & Average heart rate during 24 hours \\
\hline & SDNN & $\mathrm{ms}$ & Standard deviation of NN intervals \\
\hline & RMSSD & $\mathrm{ms}$ & $\begin{array}{l}\text { Square root of the mean squared differences of successive NN } \\
\text { intervals }\end{array}$ \\
\hline & pNN50 & $\%$ & $\begin{array}{l}\text { Proportion of interval differences of successive NN intervals greater } \\
\text { than } 50 \mathrm{~ms}\end{array}$ \\
\hline \multirow{6}{*}{ HRV statistics } & LFnu-RRI & $\%$ & LF power in normalized units \\
\hline & HFnu-RRI & $\%$ & HF power in normalized units \\
\hline & VLF-RRI & $\mathrm{ms}^{2}$ & Power in very low frequency range \\
\hline & PSD-RRI & $\mathrm{ms}^{2}$ & Total power \\
\hline & LF/HF-RRI & & Ratio LF $\left[\mathrm{ms}^{2}\right] / \mathrm{HF}\left[\mathrm{ms}^{2}\right]$ \\
\hline & BRS & $\mathrm{ms} / \mathrm{mmHg}$ & $\begin{array}{l}\text { Change in interbeat interval (IBI) in milliseconds per unit change in } \\
\text { BP }\end{array}$ \\
\hline
\end{tabular}

that we do not cut off some real data that are not errors. Extreme values were easy to identify since they were usually ten times larger than the expected range. Missing values were replaced with mean value of the corresponding attribute of all instances in the corresponding class. Although this is the simplest technique, it guarantees that it will not disturb dataset statistics. Unbalanced data is the biggest problem for training neural network classifier and classifier performance evaluation. Balanced datasets were created by randomly selecting the same number of patients (200) from both classes of patients (survived and died). These are not the only techniques (and probably not the best) that could be used, but they represent common practice and they gave good results. A detailed description of all fifteen parameters used in this study is provided in Table 1 .

Some of these parameters were selected as mortality risk predictors based on the statistical analysis of previously conducted studies $[21,22]$. Based on these studies, these parameters have been chosen for ANN training in this study, in order to further investigate their predictive power. Statistical analysis was used to assess the predictive power of the selected parameters.

2.4. Statistical Analysis. Survival curve (Figure 1) was created using the Kaplan-Meier method. The primary endpoint of the study was all-cause mortality. Associations between variables and mortality were assessed using stepwise multivariate Cox regression. The proportional hazard for mortality over time was assessed with Cox regression. The regression model was built by initially including factors with significant $(P<0.05)$ univariate associations. Multivariate Cox regression (SPSS software, version 19; SPSS Inc.) was used to determine a hazard ratio (HR) that was corrected for possible confounding variables. HRs and 95\% confidence intervals (CIs) are presented; a $P$ value $\leq 0.05$ was considered to be statistically significant. These results show that selected parameters have significant predictive power for predicting all-cause mortality.

2.5. Neural Network Training. To train a neural network classifier, the neural network software Neuroph (http://neuroph .sourceforge.net/) was used (Figure 2). Neuroph is free, open source software and provides a graphical user interface and visual tools (Figure 3), which enable experimentation with different settings for neural network architectures and training.

The multilayer perceptron neural network architecture with back propagation learning algorithm was used to train a neural network. This architecture is well known for its application for classification problems. Randomly selected $70 \%$ of the dataset was used to train neural network classifiers, while the rest of it (30\%) was used for testing. This kind of dataset split is common practice in neural network training that is used to avoid overfitting the network and achieve good classification performance with data that has not been used for training [23]. The exact split ratio depends mostly on the amount of data, and the optimal ratio can be determined heuristically. The same technique has been used in other related studies [7].

Training and testing procedures were repeated multiple times to determine optimal neural network settings (number of hidden layers and neurons) and learning rule parameters. 


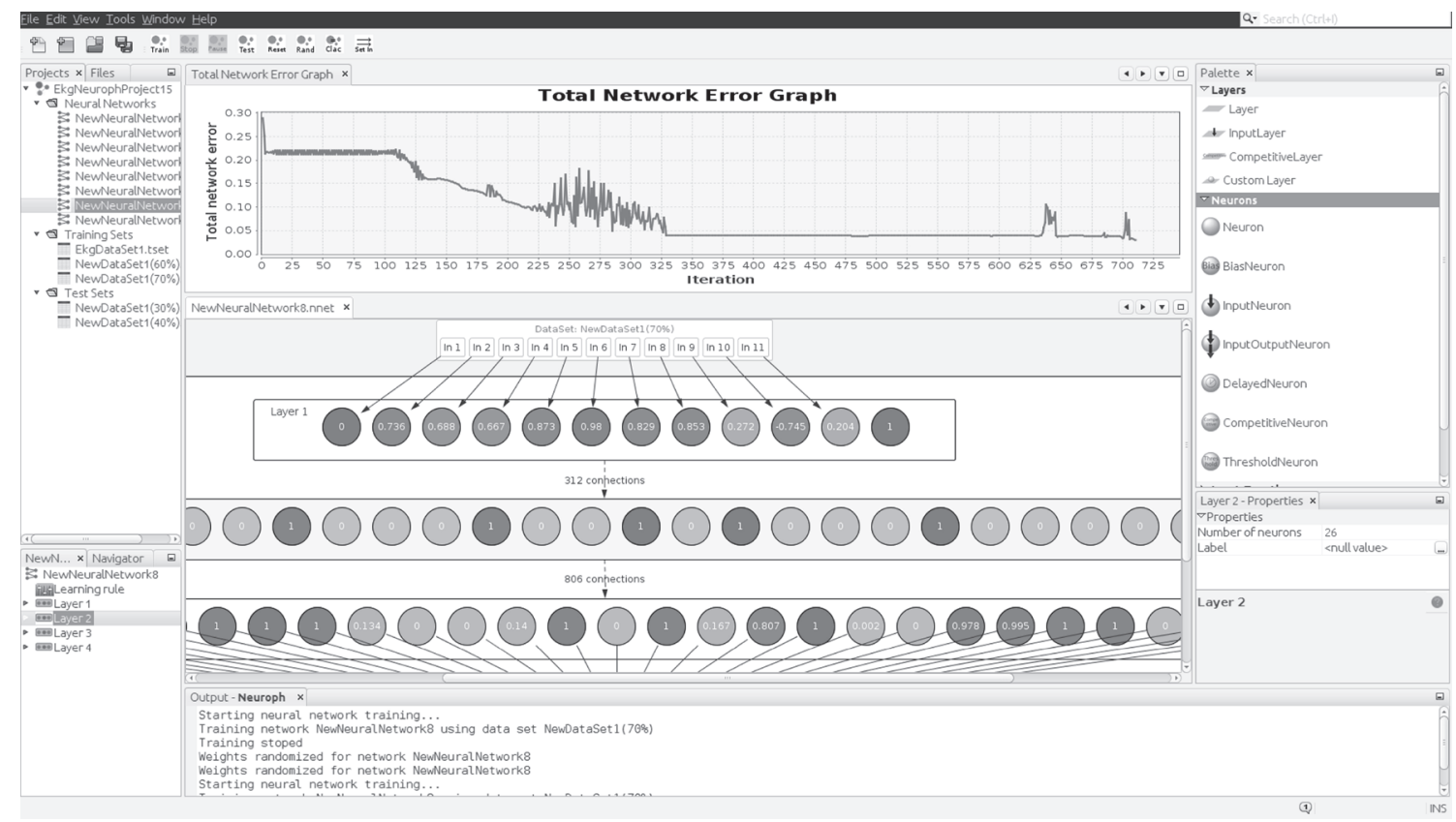

FIGURE 1: Neuroph software used for neural network training.

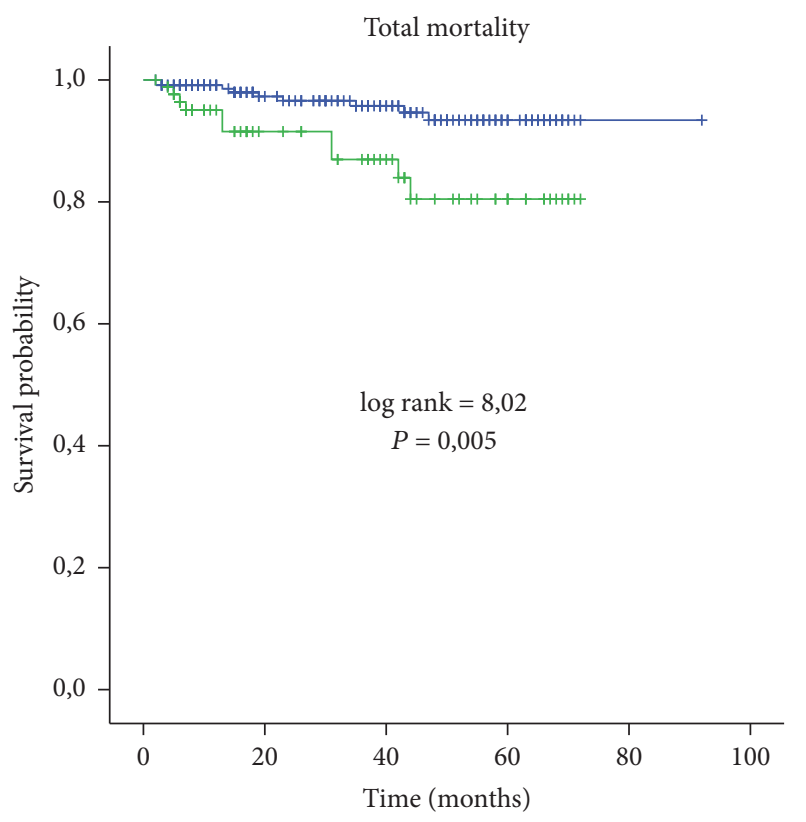

BRS et enrolment

$$
\begin{array}{ll}
\neg>5.33 & +>5.33 \text {, censored } \\
\neg \leq 5.33 & +\leq 5.33 \text {, censored }
\end{array}
$$

FIGURE 2: Kaplan-Meier survival curves for cardiac death in patients with reduced BRS at or below $5.33 \mathrm{~ms} / \mathrm{mmHg}$ in early phase after acute myocardial infarction.

\section{Results}

During a median follow-up of 3 years (range, 1.2-4.8 y), 286 $(16.77 \%)$ of the 1705 patients died from all cardiac causes. Unadjusted Kaplan-Meier survival showed worse survival for

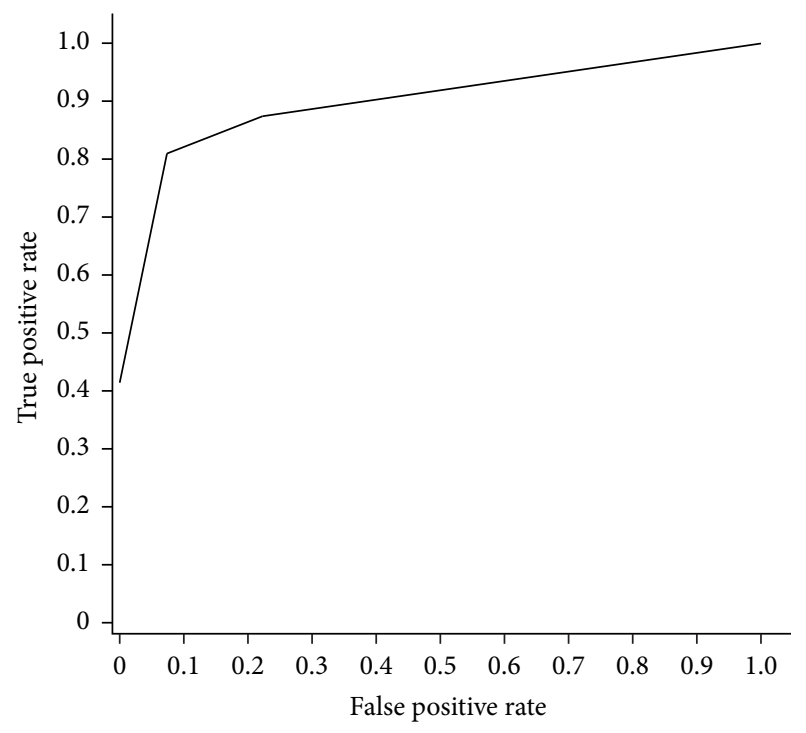

FIGURE 3: ROC curve for the neural network classifier to predict a survival status.

reduced BRS under 5,33 ms $/ \mathrm{mmHg}(P=0,005)$ (Figure 2). Univariable Cox proportional-hazards model showed statistically significant hazard ratio for the following covariates presented in Table 2, with suggested cutoff values and intervals which were used for further classification and prediction. Multivariable regression revealed significant associations of reduced BRS under 5,33 ms/mmHg (HR: 5.58, 95\% CI: $1.25-24.95, P=0.047)$ with survival. There were no statistically significant differences between the hazard ratios of either all-cause or cardiovascular mortality for LFnu-RRI, HFnu-RRI, and LF/HF-RRI. 
TABLE 2: Association of risk variables with cardiac mortality in univariable analysis.

\begin{tabular}{lccc}
\hline Method & Variable (values) & Hazard ratio, 95\% CI (univariable) & $P$ \\
\hline \multirow{4}{*}{ ECG parameters } & QT interval $(\leq 378)$ & $1.38(1.08-1.76)$ & 0.009 \\
& QTc interval $(>434)$ & $1.28(1.00-1.67)$ & 0.050 \\
& PR interval $(151-184)$ & $0.69(0.52-0.91)$ & 0.008 \\
& QRS complex $(\leq 87)$ & $0.56(0.42-0.75)$ & 0.001 \\
& P wave $(64.19-72.36)$ & $2.14(1.06-4.34)$ & 0.034 \\
\hline \multirow{2}{*}{ Time domain analysis } & Average HR $(>84.7)$ & $1.73(1.05-2.86)$ & 0.032 \\
& SDNN $(\leq 120.0)$ & $9.33(1.31-66.7)$ & 0.026 \\
& RMSSD $(>53.7)$ & $1.58(1.04-2.40)$ & 0.030 \\
HRV statistics & pNN50 $(\leq 2.7)$ & $8.84(1.14-68.56)$ & 0.037 \\
\hline
\end{tabular}

SDNN: standard deviation of all the RR intervals; RMSSD: square root of the mean of squared differences of two consecutive RR intervals; pNN50: percent of beats with consecutive RR interval difference of more than 50 milliseconds; VLF: very low frequency component of HRV; PSD: total power of HRV; HRV: heart rate variability; ECG: electrocardiogram.

TABLE 3: Classifier performance for different neural network architectures.

\begin{tabular}{lcccccc}
\hline Number & Neural network architecture & Learning Rate & Accuracy & Sensitivity & Specificity & $F$-measure \\
\hline$(1)$ & $11-13-1$ & 0.2 & 0.73 & 0.6 & 0.8 & 0.6 \\
$(2)$ & $11-15-1$ & 0.2 & 0.8 & 0.6 & 0.9 & 0.6 \\
$(3)$ & $11-30-1$ & 0.3 & 0.8 & 0.6 & 0.9 & 0.6 \\
$(4)$ & $11-15-7-1$ & 0.1 & 0.73 & 0.6 & 0.8 & 0.6 \\
$(5)$ & $11-25-30-1$ & 0.2 & 0.88 & 0.81 & 0.85 \\
$(6)$ & $11-25-40-1$ & 0.25 & 0.73 & 0.8 & 0.7 \\
\hline
\end{tabular}

During the neural network training, a number of different settings for neural network architecture were tested. For each trained model a set of standard classification performance measures were calculated using a test set. The most critical parameters for successful training and classifier performance turned out to be a number of hidden layers, a number of hidden neurons, and error threshold. The results are shown in Table 3.

All architectures had 11 input neurons which correspond to 11 input parameters and one output neuron which corresponds to a single binary output. The neural network architecture with 25 neurons in the first hidden layer and 30 neurons in the second hidden layer (number (5)) showed the best classification performance: $88 \%$ accuracy, $81 \%$ sensitivity, 93\% specificity, and $0.85 \mathrm{~F}$-measure. The Receiver Operating Characteristics (ROC) curve for the best neural network classifier (architecture number (5)) is shown in Figure 3. The area under the curve value is as follows: AUC $=0.77$.

Another critical parameter for getting good generalization ability of the network and testing results was the error threshold. The value of 0.03 for error threshold showed the best results.

\section{Discussion}

The electrocardiogram and blood pressure monitoring can be used in evaluation of patients in early phase after myocardial infarction. Over the last several years, computer-based learning models significantly improved the ability to predict adverse cardiac events [24]. The current understanding of the clinically relevant predictors in patients after AMI is reviewed in this article. Impaired baroreflex sensitivity following MI has been extensively documented in previous studies $[25,26]$. In humans, depressed BRS after myocardial infarction is a clinical predictor of increased mortality [27-29]. In our study, multivariate analysis showed that reduced value of baroreflex sensitivity below $5.33 \mathrm{~ms} / \mathrm{mmHg}$ in the observed group of patients is the only independent risk predictor of all investigated parameters.

The prediction model was built on initially included factors which were statistically significant in univariate analysis. The main disadvantage of univariate analysis is that it describes the survival with respect to the factor under investigation but necessarily ignores the impact of any others [30]. Nevertheless, it is likely that the assumptions required by the Cox model may not be satisfied because the model assumes that the underlying hazard rate is a function of independent variables, instead of the survival time [31]. Survival analysis using the Cox regression model generally shows the results based on the whole population, but it is insufficient to predict on individual level. In predictions of survival for individual patients, neural networks constitute good alternatives for classical statistical methods [32].

Although some previous studies have shown the association between prolonged QTc interval and cardiovascular mortality or all-cause mortality in the general population, other studies suggest that the relationship is not consistent 
and the risk is likely to be small $[33,34]$. In our study, univariate Cox regression analysis of individual risk predictors using ECG parameters, short-term heart-rate variability parameters, and 24-hour Holter monitoring parameters did not show statistical significance for survival in patients after myocardial infarction using fundamental analysis. Making the autonomic nervous system patterns for each parameter and its analysis for the particular value interval gave statistical significance which pointed to the advantage of using this method in statistics. One study had performed serial ECG follow-up after AMI and showed that QTc interval > $440 \mathrm{~ms}$ at hospital discharge had independent value for predicting major cardiac events [35]; another group suggested that QTc interval $>445 \mathrm{~ms}$ independently predicts all-cause death and heart failure in patients with STEMI [36]. Our study showed statistical significance for all-cause cardiac mortality in a group with QTc interval > $434 \mathrm{~ms}$. The ATRAMI study showed that low values of either heart-rate variability (SDNN $<70 \mathrm{~ms})$ or BRS $(<3.0 \mathrm{~ms} / \mathrm{mm} \mathrm{Hg})$ are a significant multivariate risk of cardiac mortality [37] compared to our study where reduced $\mathrm{SDNN}<120 \mathrm{~ms}$ suggested higher risk. The predictive value of a variety of parameters has been assessed using ANN. Adding the artificial neural networks in data analysis in patients with acute myocardial infarction, the survival methodology was significantly improved, confirming that this model is fully applied and accurate in risk stratification and follow-up. Using standard statistical models we have concluded that the group with higher cardiovascular risk has sympathetic hyperactivity. In accordance with the results, therapy which includes adrenergic blockade should be proposed (beta blockers, ACE inhibitors, etc.). Similar results have been published in other studies [38, 39]. The ANN showed significantly better results in predicting survival of patients than the other models commonly used in different studies [40-42].

The proposed neural network classifier showed good performance for survival prediction, although the sample size was insufficient to ensure the statistical satisfaction. Part of the problem in preparing the data is incomplete and erroneous data, which were manually resolved by calculating corresponding values or removing samples.

The neural network training required a lot of experimenting to figure out the best architecture (number of hidden layers and neurons) and achieve the optimal classification performance. The good results were already obtained with one hidden layer with 15 hidden neurons; however increasing that number to 30 hidden neurons did not give any improvement in classification performance. So the conclusion was that using a single hidden layer with more than 15 neurons increases computational requirements, without increasing classifier performance, and probably leads to overfitting the model.

Adding another hidden layer and using the architecture with 11-15-7-1 neurons in layers, respectively, also did not give any improvements. So the conclusion was that adding one more layer on top of the first hidden layer also does not lead to classifier performance improvement.

Using architecture number 5, with 11-25-30-1 neurons in layers, gave the best classification performance. It is also interesting to note that adding more hidden neurons to second hidden layer (architecture (6)) gave worse results than architecture (5) (almost as architecture (1)). These results show that using more hidden neurons and layers does not bring improvement in classifier performance and that it is one of the critical parameters that needs to be further investigated for this type of applications. The optimal architecture and classification performance largely depends on which input parameters are used. The results showed that the statistical analysis can indicate a reliable choice of input parameters.

\section{Limitations}

The present study has some limitations. One of the limitations in this study considers the signal required for heart-rate variability analysis. In order to obtain clear signal, the samples with any noise or arrhythmias were eliminated, which reduced a group of patients with higher cardiovascular risk after myocardial infarction. To overcome the limitations, it is necessary to conduct research on a larger group of patients. ECG parameters like QTc intervals at discharge and after discharge are not measured or analyzed, and it is unknown whether they will have better predictive value. During post-MI period, assessment of cardiovascular risk parameters should be repeated multiple times. Another limitation is related to black-box nature of algorithm. At this point, it is hard to explain and debug results, as well as to understand possible different results. Making bigger dataset with more input variables during longer time of follow-up would provide more precise conclusion especially about very complex mechanisms involved in pathogenesis of cardiovascular diseases. The selection of risk factors which will be used as inputs for the neural network should be derived through a feature selection procedure on the training set, within the ANN cross-validation procedure. Further investigation needs to be established, but a good survival predictor must be able to deal with these obstacles.

\section{Conclusion}

This study proposed a survival status predictor based on ANN classifier trained with data obtained from HRV analysis. The trained ANNs achieved satisfying contribution in the prediction of outcome in patients with higher risk after MI. The study outlined the procedure for building neural network based clinical decision-making algorithm, including data preprocessing, attribute selection, neural network tuning, and performance evaluation. Classification based on the autonomic nervous system patterns can reliably indicate the individuals with a higher risk. This knowledge is crucial for making decisions about further observation and treatment.

Classifier performance largely depends on the input parameters which are used for training the ANN and number of hidden layers and neurons. Good candidates for input parameters for ANN training can be determined using statistical analyses and Kaplan-Meier survival curves for cardiac death. The optimal neural network architecture (number of hidden layers and neurons) can be determined experimentally by systematically adding hidden neurons and layers 
and observing the classifier performance for each setting. The performance of the proposed method should be further investigated using databases from other sources.

\section{Conflicts of Interest}

The authors declare that there are no conflicts of interest regarding the publication of this article.

\section{Acknowledgments}

This work is supported by the Ministry of Education, Science and Technological Development of Serbia under Grants TP32040 and III 45003.

\section{References}

[1] J. Lette, B. W. Colletti, M. Cerino et al., "Artificial intelligence versus logistic regression statistical modelling to predict cardiac complications after noncardiac surgery," Clinical Cardiology, vol. 17, no. 11, pp. 609-614, 1994.

[2] S. C. Smith Jr., J. Allen, S. N. Blair et al., "AHA/ACC guidelines for secondary prevention for patients with coronary and other atherosclerotic vascular disease: 2006 update-endorsed by the national heart, lung, and blood institute," Circulation, vol. 113, no. 19, pp. 2363-2372, 2006.

[3] M. O. Sweeney, M. S. Wathen, K. Volosin et al., "Appropriate and inappropriate ventricular therapies, quality of life, and mortality among primary and secondary prevention implantable cardioverter defibrillator patients: results from the pacing fast VT REduces shock ThErapies (PainFREE Rx II) trial," Circulation, vol. 111, no. 22, pp. 2898-2905, 2005.

[4] M. T. La Rovere, G. D. Pinna, S. H. Hohnloser et al., "Baroreflex sensitivity and heart rate variability in the identification of patients at risk for life-threatening arrhythmias," Circulation, vol. 103, no. 16, pp. 2072-2077, 2001.

[5] F. M. Merchant, T. Quest, A. R. Leon, and M. F. El-Chami, "Implantable cardioverter-defibrillators at end of battery life: opportunities for risk (Re)-stratification in ICD recipients," Journal of the American College of Cardiology, vol. 67, no. 4, pp. 435-444, 2016.

[6] P. E. Puddu and A. Menotti, "Artificial neural network versus multiple logistic function to predict 25-year coronary heart disease mortality in the Seven Countries Study," European Journal of Cardiovascular Prevention and Rehabilitation, vol. 16, no. 5, pp. 583-591, 2009.

[7] S. Joo, K.-J. Choi, and S.-J. Huh, "Prediction of spontaneous ventricular tachyarrhythmia by an artificial neural network using parameters gleaned from short-term heart rate variability," Expert Systems with Applications, vol. 39, no. 3, pp. 38623866, 2012.

[8] R. Bigi, D. Gregori, L. Cortigiani, A. Desideri, F. A. Chiarotto, and G. M. Toffolo, "Artificial neural networks and robust Bayesian classifiers for risk stratification following uncomplicated myocardial infarction," International Journal of Cardiology, vol. 101, no. 3, pp. 481-487, 2005.

[9] Z. Sevarac, B. Milovanovic, and T. Gligorijevic, "Follow up and risk assessment using artificial neural networks in patients with myocardial infarction," in Proceedings of the 7th International Symposium on Noninvasive Electrocardiology (NEUROCARD '16), Belgrade, Serbia, October 2016.
[10] D. V. Exner, "Noninvasive risk stratification after myocardial infarction: rationale, current evidence and the need for definitive trials," Canadian Journal of Cardiology, vol. 25, supplement A, pp. 21A-27A, 2009.

[11] T. Huebner, M. Goernig, M. Schuepbach et al., "Electrocardiologic and related methods of non-invasive detection and risk stratification in myocardial ischemia: state of the art and perspectives," German Medical Science, vol. 8, article Doc27, 2010.

[12] J. V. Tu, "Advantages and disadvantages of using artificial neural networks versus logistic regression for predicting medical outcomes," Journal of Clinical Epidemiology, vol. 49, no. 11, pp. 1225-1231, 1996.

[13] B. Eftekhar, K. Mohammad, H. E. Ardebili, M. Ghodsi, and E. Ketabchi, "Comparison of artificial neural network and logistic regression models for prediction of mortality in head trauma based on initial clinical data," BMC Medical Informatics and Decision Making, vol. 5, no. 1, article 3, 2005.

[14] L. Bottaci, P. J. Drew, J. E. Hartley et al., "Artificial neural networks applied to outcome prediction for colorectal cancer patients in separate institutions," The Lancet, vol. 350, no. 9076, pp. 469-472, 1997.

[15] P. E. Puddu and A. Menotti, "Artificial neural networks versus proportional hazards Cox models to predict 45-year all-cause mortality in the Italian Rural Areas of the Seven Countries Study," BMC Medical Research Methodology, vol. 12, article 100, 2012.

[16] M. Kusy, B. Obrzut, and J. Kluska, "Application of gene expression programming and neural networks to predict adverse events of radical hysterectomy in cervical cancer patients," Medical and Biological Engineering and Computing, vol. 51, no. 12, pp. 1357-1365, 2013.

[17] F. E. Ahmed, "Artificial neural networks for diagnosis and survival prediction in colon cancer," Molecular Cancer, vol. 4, article 29, 2005.

[18] G. Parati, G. Ongaro, G. Bilo et al., "Non-invasive beat-to-beat blood pressure monitoring: new developments," Blood Pressure Monitoring, vol. 8, no. 1, pp. 31-36, 2003.

[19] G. Parati, M. DiRienzo, and G. Mancia, "How to measure baroreflex sensitivity: from the cardiovascular laboratory to daily life," Journal of Hypertension, vol. 18, no. 1, pp. 7-19, 2000.

[20] G. Gratze, R. Rudnicki, W. Urban, H. Mayer, A. Schlögl, and F. Skrabal, "Hemodynamic and autonomic changes induced by Ironman: prediction of competition time by blood pressure variability," Journal of Applied Physiology, vol. 99, no. 5, pp. 17281735, 2005.

[21] W. S. Choi, Y. Cho, N. Y. Kim et al., "Prognostic value of standard electrocardiographic parameters for predicting major adverse cardiac events after acute myocardial infarction," Circulation, vol. 122, supplement 21, pp. A9026-A9026, 2010.

[22] E. S. Williams, K. L. Thomas, S. Broderick et al., "Race and gender variation in the QT interval and its association with mortality in patients with coronary artery disease: results from the Duke Databank for Cardiovascular Disease (DDCD)," American Heart Journal, vol. 164, no. 3, pp. 434-441, 2012.

[23] M. C. Bishop, Neural Networks for Pattern Recognition, Clarendon Press, Oxford, UK, 1995.

[24] C. Castaneda, K. Nalley, C. Mannion et al., "Clinical decision support systems for improving diagnostic accuracy and achieving precision medicine," Journal of Clinical Bioinformatics, vol. 5, no. 1, p. 4, 2015. 
[25] O. Kjellgren and J. A. Gomes, "Heart rate variability and baroreflex sensitivity in myocardial infarction," American Heart Journal, vol. 125, no. 1, pp. 204-215, 1993.

[26] A. J. Minisi, T. B. Nashed, and M. S. Quinn, "Regional left ventricular deafferentation increases baroreflex sensitivity following myocardial infarction," Cardiovascular Research, vol. 58, no. 1, pp. 136-141, 2003.

[27] A. D. Michaels and N. Goldschlager, "Risk stratification after acute myocardial infarction in the reperfusion era," Progress in Cardiovascular Diseases, vol. 42, no. 4, pp. 273-309, 2000.

[28] G. M. De Ferrari, A. Sanzo, A. Bertoletti, G. Specchia, E. Vanoli, and P. J. Schwartz, "Baroreflex sensitivity predicts long-term cardiovascular mortality after myocardial infarction even in patients with preserved left ventricular function," Journal of the American College of Cardiology, vol. 50, no. 24, pp. 2285-2290, 2007.

[29] C. M. Jones, M. S. Quinn, and A. J. Minisi, "Reflex control of sympathetic outflow and depressed baroreflex sensitivity following myocardial infarction," Autonomic Neuroscience: Basic and Clinical, vol. 141, no. 1-2, pp. 46-53, 2008.

[30] M. Babińska, J. Chudek, E. Chełmecka, M. Janik, K. Klimek, and A. Owczarek, "Limitations of Cox proportional hazards analysis in mortality prediction of patients with acute coronary syndrome," Studies in Logic, Grammar and Rhetoric, vol. 43, no. 56, pp. 33-48, 2015.

[31] M. J. Bradburn, T. G. Clark, S. B. Love, and D. G. Altman, "Survival analysis part II: multivariate data analysis-an introduction to concepts and methods," British Journal of Cancer, vol. 89, no. 3, pp. 431-436, 2003.

[32] L. Ohno-Machado, "A comparison of Cox proportional hazards and artificial neural network models for medical prognosis," Computers in Biology and Medicine, vol. 27, no. 1, pp. 55-65, 1997.

[33] S. M. Straus, J. A. Kors, M. L. de Bruin et al., "Prolonged QTc interval and risk of sudden cardiac death in a population of older adults," Journal of the American College of Cardiology, vol. 47, no. 2, pp. 362-367, 2006.

[34] A. Montanez, J. N. Ruskin, P. R. Hebert, G. A. Lamas, and C. H. Hennekens, "Prolonged QTc interval and risks of total and cardiovascular mortality and sudden death in the general population: a review and qualitative overview of the prospective cohort studies," Archives of Internal Medicine, vol. 164, no. 9, pp. 943-948, 2004.

[35] S. Ahnve, "QT interval prolongation in acute myocardial infarction," European Heart Journal, vol. 6, supplement D, pp. 85-95, 1985.

[36] J. F. Lin, S. Y. Hsu, S. Wu et al., "QT interval independently predicts mortality and heart failure in patients with ST-elevation myocardial infarction," International Journal of Medical Sciences, vol. 12, no. 12, article 968, 2015.

[37] M. T. La Rovere, J. T. Bigger Jr., F. I. Marcus, A. Mortara, and P. J. Schwartz, "Baroreflex sensitivity and heart-rate variability in prediction of total cardiac mortality after myocardial infarction," The Lancet, vol. 351, no. 9101, pp. 478-484, 1998.

[38] L. N. Graham, P. A. Smith, J. B. Stoker, A. F. Mackintosh, and D. A. S. G. Mary, "Time course of sympathetic neural hyperactivity after uncomplicated acute myocardial infarction," Circulation, vol. 106, no. 7, pp. 793-797, 2002.

[39] B. S. Huang and F. H. H. Leenen, "The brain renin-angiotensinaldosterone system: a major mechanism for sympathetic hyperactivity and left ventricular remodeling and dysfunction after myocardial infarction," Current Heart Failure Reports, vol. 6, no. 2, pp. 81-88, 2009.
[40] G. Qiao, J. Li, A. Huang, Z. Yan, W.-Y. Lau, and F. Shen, "An artificial neural networking model for the prediction of post-hepatectomy survival of patients with early hepatocellular carcinoma," Journal of Gastroenterology and Hepatology, vol. 29, no. 12, pp. 2014-2020, 2014.

[41] C. G. Raji and S. S. Vinod Chandra, "Graft survival prediction in liver transplantation using artificial neural network models," Journal of Computational Science, vol. 16, pp. 72-78, 2016.

[42] J. H. Peng, Y. J. Fang, C. X. Li et al., "A scoring system based on artificial neural network for predicting 10-year survival in stage II A colon cancer patients after radical surgery," Oncotarget, vol. 7, no. 16, Article ID 22939, 2016. 


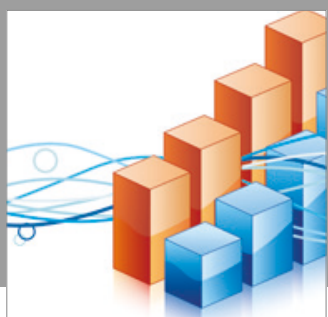

Advances in

Operations Research

vatersals

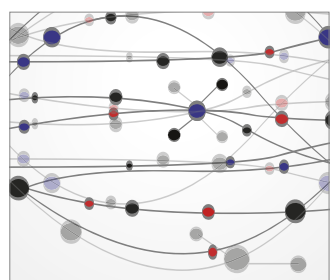

\section{The Scientific} World Journal
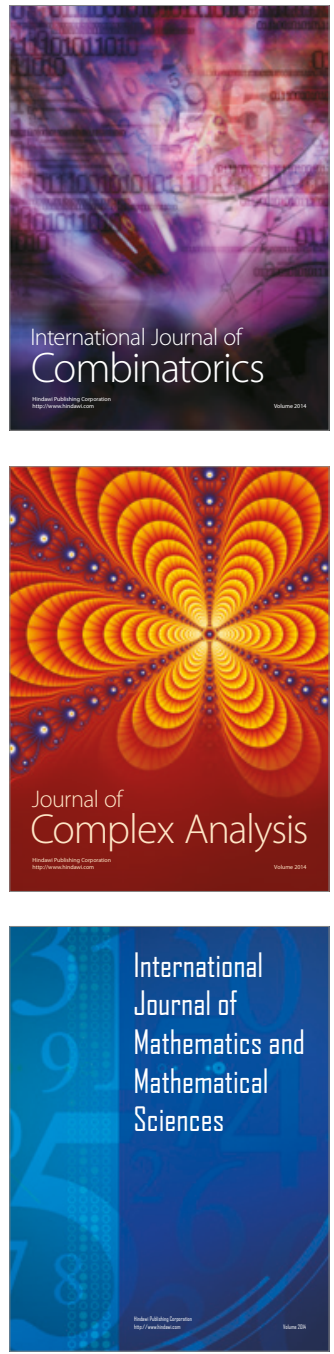
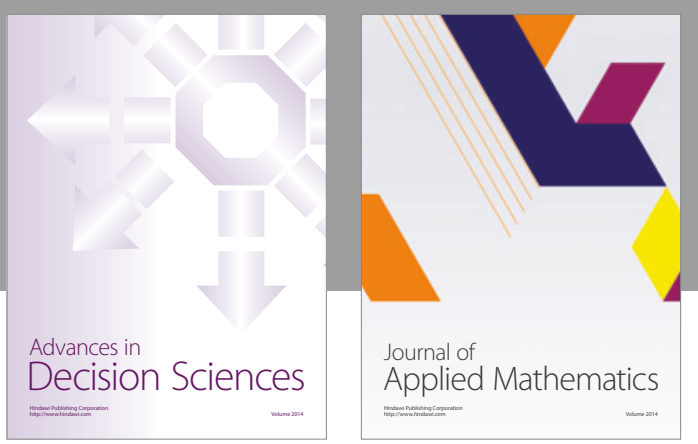

Algebra

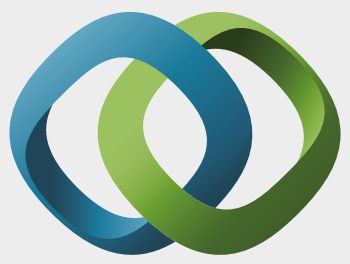

\section{Hindawi}

Submit your manuscripts at

https://www.hindawi.com
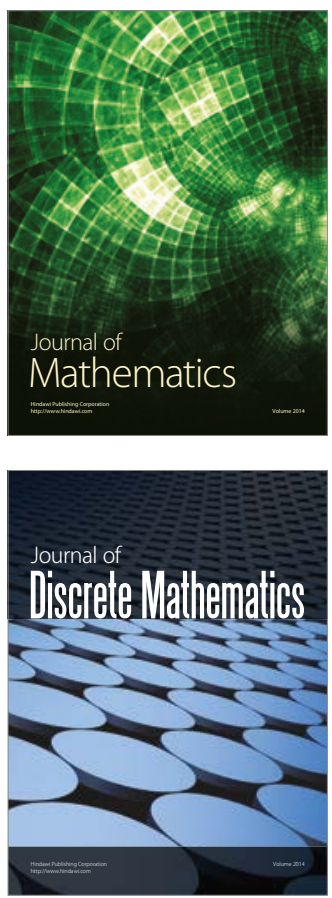

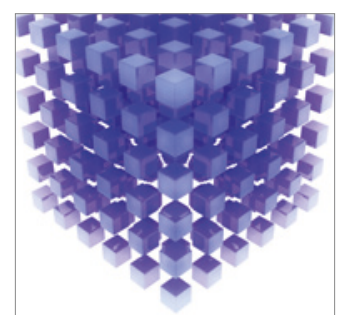

Mathematical Problems in Engineering
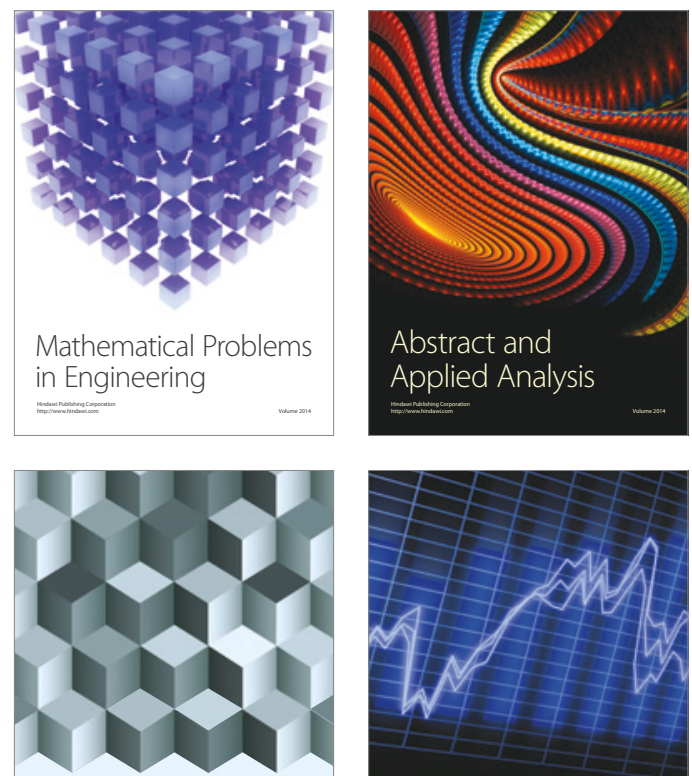

Journal of

Function Spaces

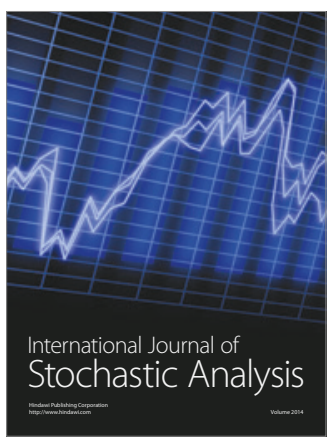

Probability and Statistics
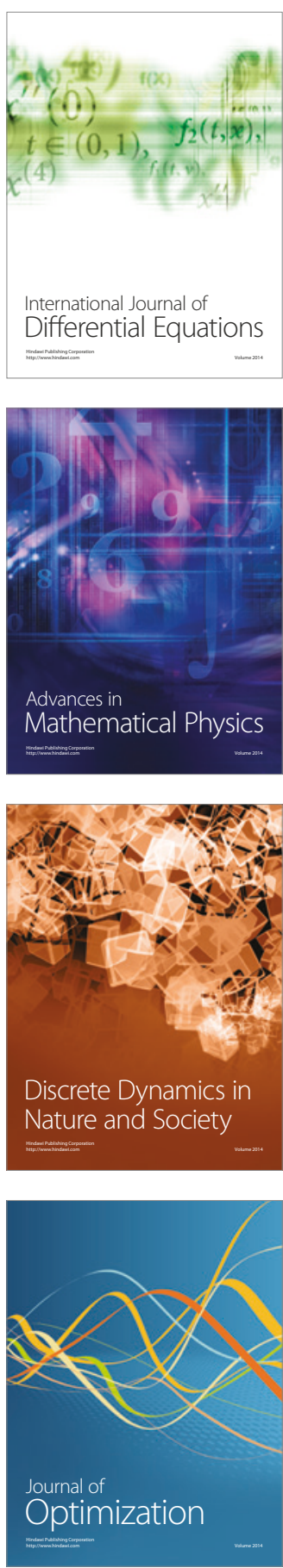